\title{
Tensions interethniques et confrontations de croyances dans la littérature mansie des années 1930
}

Deux chasseurs de Pantelejmon Evrin

Interethnic tensions and religious confrontations in Western Siberia in the 1930s: Pantelejmon Evrin and Two Hunters

Rahvustevahelised pinged ja usundite kokkupõrked Lääne-Siberis 1930. aastatel: Panteleimon Jevrin ja „Kaks jahimeest"

\section{Eva Toulouze}

\section{(2) OpenEdition}

\section{Édition électronique}

URL : https://journals.openedition.org/efo/76

DOI : $10.4000 /$ efo. 76

ISSN : 2275-1947

Éditeur

INALCO

\section{Édition imprimée}

Date de publication : 1 janvier 2011

ISBN : 978-2-296-96641-3

ISSN : 0071-2051

\section{Référence électronique}

Eva Toulouze, «Tensions interethniques et confrontations de croyances dans la littérature mansie des années 1930 », Études finno-ougriennes [En ligne], 43 | 2011, mis en ligne le 19 mars 2014, consulté le 08 juillet 2021. URL : http://journals.openedition.org/efo/76 ; DOI : https://doi.org/10.4000/efo.76

Ce document a été généré automatiquement le 8 juillet 2021.

\section{(ब) (1) \&}

Études finno-ougriennes est mis à disposition selon les termes de la Licence Creative Commons Attribution - Pas d'Utilisation Commerciale 4.0 International. 


\title{
Tensions interethniques et confrontations de croyances dans la littérature mansie des années 1930
}

\author{
Deux chasseurs de Pantelejmon Evrin \\ Interethnic tensions and religious confrontations in Western Siberia in the 1930s: \\ Pantelejmon Evrin and Two Hunters \\ Rahvustevahelised pinged ja usundite kokkupõrked Lääne-Siberis 1930. aastatel: \\ Panteleimon Jevrin ja „Kaks jahimeest “
}

\section{Eva Toulouze}

1 Le petit ${ }^{1}$ peuple mansi 11432 personnes au recensement de $2002^{2}$ - a connu en littérature un sort tout à fait étonnant : il a donné naissance, dans la deuxième moitié $\mathrm{du} \mathrm{xx}^{\mathrm{e}}$ siècle, à un poète d'exception qui s'est fait connaître dans tout cet immense pays qu'était à l'époque l'Union soviétique, voire au-delà. Juvan Šestalov (1937-2011) a, certes, bénéficié des orientations de la politique culturelle officielle, qui tendait à repérer et à promouvoir des intellectuels « dans la ligne » issus des nationalités, et dont au moins un par groupe ethnique bénéficiait d'un large soutien éditorial. Vue avec le recul, cette position d'écrivain "officiel », qui a parfois récompensé des personnalités médiocres, a permis également de faire ressortir de véritables talents. Pour ce qui est des peuples du Nord, la question d'être ou de ne pas être «dans la ligne » ne se posait pas sérieusement. Issues d'initiatives concrètes prises dans les années 1920-1930 par le pouvoir soviétique, les intelligentsias de ces communautés lui demeuraient fondamentalement et sincèrement fidèles. C'est par une écriture originale et puissante que Juvan Šestalov a fait largement connaître l'existence même de son peuple.

2 Mais s'il est vrai que Šestalov a réussi une étonnante percée, il ne faut pas oublier pour autant les autres noms de la courte histoire de la littérature mansie. Il faut mentionner la brève célébrité d'un texte quasi autobiographique de Matra Vahruševa ${ }^{3}$ (1918-2000), que je me permets de citer en reprenant son prénom de naissance, en souvenir d'une conversation à Saint-Pétersbourg en 1997 où elle m'a expliqué comment ce prénom 
avait été transformé à l'école en Matrjona. Ou encore le patrimoine légué par Anna Kon'kova ( ? - 1999), la "grand-mère Anné » de ses contes, qui, elle aussi disparue comme Vahruševa à la fin du $\mathrm{xx}^{\mathrm{e}}$ siècle, laisse un souvenir d'une grande limpidité. $\mathrm{Ou}$ encore, toujours heureusement parmi nous, le poète Andrej Tarhanov (né en 1936), qui écrit désormais en russe des poèmes d'un lyrisme touchant, non sans influence du christianisme. Et puis, il est possible de remonter aux origines : la littérature mansie a aussi son père fondateur, présent dès l'émergence des premières œuvres des littératures du Nord. Pantelejmon Čejmetov ${ }^{4}$, qui écrit sous le nom de plume d'Evrin ${ }^{5}$, en pose en effet la première pierre en 1940, avec un récit intitulé Deux chasseurs ${ }^{6}$, écrit en mansi et traduit en russe par V. Naumova.

3 Les œuvres de cette première période des littératures des peuples du Nord sont, de manière générale, marquées en même temps par l'enthousiasme de pionniers de leurs auteurs et par leur inexpérience. Rappelons le contexte de leur émergence : la volonté délibérée des autorités de constituer une couche d'autochtones instruits, capables de servir de médiateurs et de porter les valeurs de la vie nouvelle, avait abouti d'une part à la formation, dans des institutions ad hoc, de quelques promotions issues des peuples du Nord et, d'autre part, à la mise en route du processus de création de langues littéraires (Toulouze 1999). Largement associée à ce dernier, cette première génération d'intellectuels vécut une fabuleuse aventure, convaincue qu'elle était d'ouvrir la voie à un avenir radieux pour les peuples dont ses représentants étaient issus. Ceci explique l'enthousiasme qui se sent dans les pages des premiers textes: la découverte d'un monde nouveau, le sentiment de faire œuvre historique. En même temps, l'aventure de ces jeunes intellectuels ne va pas sans difficulté, qu'ils écrivent en russe ou dans leur langue. En russe, il s'agit de s'exprimer dans une langue étrangère, apprise pour certains déjà à l'âge adulte, porteuse d'une culture très différente, et de l'utiliser pour transmettre des réalités qui lui étaient étrangères. $\mathrm{Si}$, à l'époque, tous maîtrisaient réellement leur langue maternelle, s'en servir pour écrire n'était guère tâche facile. Ces langues n'avaient en effet jamais servi à l'écriture. Les premiers "écriveurs » défrichent ainsi un terrain entièrement vierge et ont pour mission ni plus ni moins de donner à ces langues une fonction nouvelle. 


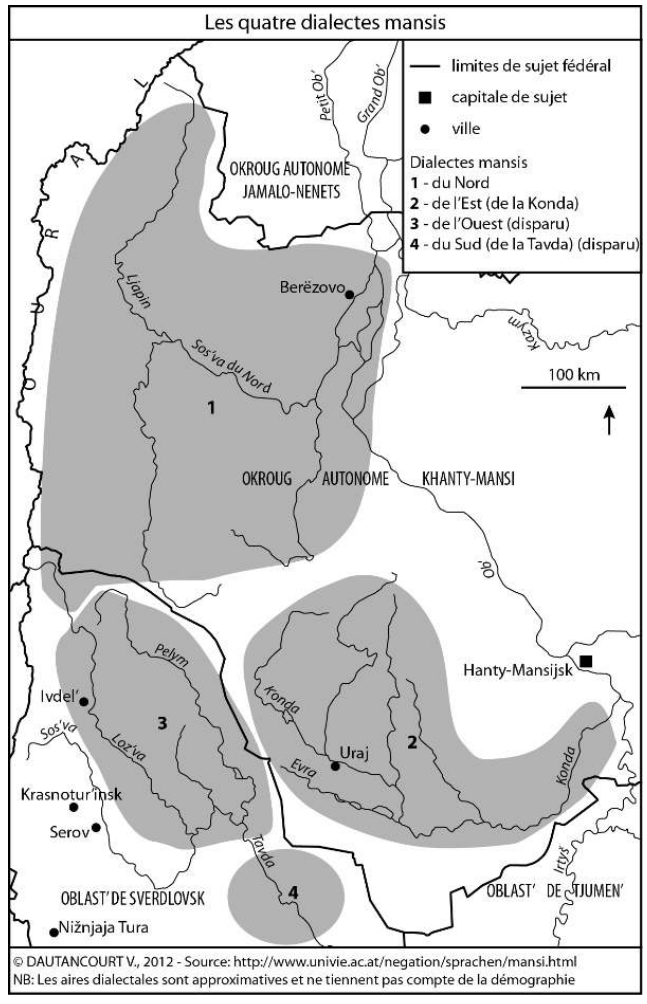

4 Nous savons que leur première aspiration aurait été de consigner les contes qui leur étaient familiers (Ogryzko 2002, p. 5). Leurs enseignants ont pour leur part contrecarré cette tendance. Nous ne pouvons que reconstruire leurs motivations. Sans doute, l'attention portée au folklore en général et aux contes en particulier aurait-elle éloigné la littérature du canon de l'époque, celui du réalisme socialiste. Il faut bien reconnaître, pourtant, que la jeune littérature des peuples du Nord n'est pas examinée et critiquée à l'aune du réalisme socialiste. Elle est manifestement considérée encore comme un exercice, comme une expression encore semi-ethnographique à ne pas confondre avec la littérature au sens noble du terme. Surtout, je pense que l'une des tâches de ces enseignants était non seulement de tester les langues littéraires comme outil d'expression, mais aussi d'accumuler des informations sur des peuples connus jusque-là uniquement par quelques chercheurs spécialisés. Pour changer leur vie, encore fallait-il savoir un minimum en quoi elle consistait. L'esprit pragmatique accordait dans ce contexte plus d'importance à l'autobiographie, au souvenir, à la description quasiment ethnographique qu'à la notation de l'héritage oral. Par ailleurs, il est probable que les enseignants de Leningrad se sont eux-mêmes sentis plus à l'aise à travailler sur des textes issus d'une réalité somme toute palpable, plutôt que de se confronter à des écrits reflétant un univers mental encore ésotérique, une langue métaphorique. Toujours estil que les thèmes donnés à ces étudiants les ont amenés à mettre en paroles leur vécu dans leur contrée d'origine et comportent une forte dimension autobiographique. C'est le cas non seulement des travaux d'école qui nous sont parvenus grâce aux numéros de la revue La toundra et la taïga ${ }^{7}$, publiée entre 1928 et 1933 en russe à Leningrad, ou par les textes du recueil Sur notre vie $e^{8}$ daté de 1929 , mais aussi des premières œuvres par exemple des auteurs nénètses : ainsi, «Sur l'̂̂le " ", repris sous le titre "Comment nous vivions sous le tsar ${ }^{10}$ » dans une revue d'actualité, est un texte explicite de Nikolaj Vylka ; même dans le récit d'Anton Pyrerka Le fils cadet de Vedo ${ }^{11}$, il n'est pas difficile de lire en filigrane l'expérience vécue de l'auteur. 
5 Or la première œuvre de la littérature mansie se distingue nettement de cette orientation et mérite une attention plus soutenue. Elle a été écrite en mansi de la Konda, huit ans après que le mansi a été officiellement doté d'une langue littéraire. Mais il est frappant de noter que ce n'est pas dans cette variante officielle, qui reposait sur les dialectes du Nord, qu'Evrin a rédigé son récit, mais dans la langue qui lui est proche, celle parlée dans sa région, à savoir le dialecte de la Konda. Longtemps, cette œuvre est restée pratiquement inconnue. En dehors de Russie, même les meilleurs spécialistes du monde mansi en ont longtemps ignoré l'existence : c'est ainsi qu'elle ne figure pas dans le tour d'horizon détaillé fait par Béla Kálmán en 1962 (Kálmán 1962, p. 132-133) Je vais m'arrêter un moment sur son auteur, puis sur les traits du récit qui me paraissent dignes d'attention.

\section{Une personnalité peu connue}

6 Les quelques informations précises que nous avons sur sa vie proviennent de deux sources: un article de Kuzakova, chercheuse mansie cousine de l'écrivain, et les assertions d'Ogryzko, présentées sous diverses formes. Malheureusement, nous ignorons l'origine de ses données - qui sont par ailleurs fort précises et que nous croyons fiables -, car le journaliste russe écrit dans un genre qui ne requiert pas l'explicitation de toutes les sources.

7 Celui qui écrira sous le nom d'Evrin était effectivement issu de la région d'Evra, berceau également de la future conteuse Anna Kon'kova. Ce n'est sans doute pas un hasard si cette région, ainsi que celle de la Konda, au sud du territoire mansi, ont été des foyers de culture particulièrement vivaces. En tout cas, de ce qu'il est convenu d'appeler culture au sens européen du terme : un monastère avait été fondé sur les rives de la Konda et, dès l'époque tsariste, avait fait des tentatives d'alphabétisation des autochtones convertis. Même si cette activité n'a pas eu de résultats notables en soi, elle a certainement contribué à familiariser la population avec l'écrit. En même temps, la pénétration russe a investi avant les autres cette zone à la nature moins rude que celle des aires plus septentrionales, et, sous son influence, la population autochtone s'est fixée de telle sorte qu'au début du $\mathrm{xx}^{\mathrm{e}}$ siècle les Mansis de la Konda étaient pour l'essentiel des agriculteurs sédentaires. Les villages étaient, semble-t-il, assez riches. La famille de Pantelejmon Evrin aussi (Kuzakova 1997, p. 30). Elle descendait d'un ancien clan mansi, attesté sur les lieux dès le début du XIX siècle (Kuzakova 1992, p. 39).

Le père de l'écrivain, un pêcheur, avait été à l'origine de la création de l'école du village d'Evra, et deux de ses cinq fils prendront le chemin de l'instruction. C'est en tant que kolkhozien et que komsomol que Pantelejmon Čejmetov est amené à suivre des cours de préparation dans la capitale régionale (à l'époque Ostiako-Vogul'sk, aujourd'hui Khanty-Mansijsk) avant de partir, en 1934, faire ses études à l'Institut des peuples du Nord à Leningrad (Naumova 1940, p. 50). Il semblerait qu'en partant, le jeune Mansi ait emporté quelques objets attributs du culte à offrir au musée d'ethnographie de Leningrad (ibid.). Ogryzko, mi-facétieux mi-sérieux, propose même d'y voir la raison des malheurs qui se sont acharnés sur lui (Ogryzko 1999, p. 398)...

9 Au cours de ses études, il se marie avec une étudiante khantye issue de la même région que lui, et le jeune couple aura une fille née en 1938 (Kuzakova 1997, p. 30). En même temps, les épreuves ne leur sont pas épargnées. Comme les autres premières générations d'étudiants des peuples du Nord à Leningrad, leurs années de formation 
coïncident avec le lancement des mesures radicales de collectivisation. Or la famille Čejmetov vit dans l'abondance: elle est la première visée par les mesures de dékoulakisation, et Pantelejmon en est directement victime. Il est en effet exclu de l'Institut des peuples du Nord. De retour dans sa région, il est nommé président de kolkhoze, avant de parvenir à blanchir son nom et celui de sa famille, et de réintégrer l'Institut (Ogryzko 1999, p. 397). Il y est manifestement populaire : au milieu des années 1930, il participe au tournage d'un film dans lequel il chante en s'accompagnant à l'accordéon (ibid.).

10 Parti au front finlandais comme volontaire, Evrin y est blessé, et ce ne sera pas la seule fois au cours de la guerre, jusqu'à sa démobilisation en 1944. Là, il est affecté à la direction du NKVD de Tjumen'. C'est à cette époque que remontent les dernières informations que nous avons sur sa vie : ayant découvert sa maîtresse avec un homme, il la tue, mais rate son suicide et est condamné à une peine de détention. Nous ne savons plus rien de sa vie, pas même la date de sa mort (Ogryzko 1999, p. 398). Il semble bien, en tout cas, que la guerre ait conduit sa vie à une série de catastrophes, depuis les blessures jusqu'à la tragédie finale. D'ailleurs, presque tous ses frères sont morts à la guerre (Kuzakova 1997, p. 30).

\section{Une véritable œuvre de fiction}

Dans le contexte des toutes premières œuvres en prose, le point à noter d'emblée est qu'Evrin produit réellement une œuvre de fiction. Son récit se distingue par là des autres textes originaires de Sibérie occidentale et de l'aire nénètse, caractérisés, comme je l'ai mentionné ci-dessus, par l'autobiographisme direct ou indirect. Dans le récit d'Evrin, nous trouvons, certes toute sa maîtrise de l'univers mental des Mansis, qui provient de son expérience de vie. Mais le jeune auteur met en scène des personnages par nature différents de lui : un vieux Mansi et un jeune Russe. Il révèle par là son aptitude à élaborer sa propre expérience du monde en œuvre littéraire. Par ailleurs, le sujet choisi ne fait pas appel à l'arsenal disponible des récits issus de l'oralité : le récit n'est pas un conte transposé, même si dans les péripéties de l'intrigue certains éléments peuvent rappeler de loin les récits de combat héroïque de l'homme et de l'ours. C'est dans la vie qu'Evrin va chercher les personnages de sa fiction, qui relève du genre du récit édifiant.

En effet, comme l'essentiel de la littérature de l'époque, le récit d'Evrin est un récit à message : le problème exposé dans les premières pages trouve sa solution à la fin du récit. Le vieux chasseur mansi Tyrpimij ${ }^{12}$ résiste à la pression de ses enfants, de sa femme, du village tout entier et refuse d'adhérer au kolkhoze. Au début du récit, il est pourtant hésitant. Les péripéties relatées dans le texte l'amènent, au terme de l'histoire, à prendre finalement la décision tant attendue. Cette problématique, elle, n'a rien d'original: elle correspond à l'esprit de l'époque et est, encore aujourd'hui, d'actualité. On peut se demander si la situation qui sert de toile de fond à l'action correspond à la réalité : on a l'impression que l'entourage du vieux Tyrpimij se montre particulièrement tolérant et lui laisse le temps de décider lui-même de ses actes. Or nous savons que la collectivisation s'est faite de manière générale plutôt sous la contrainte qu'en partant du libre arbitre des populations. Peu importe : il est question ici de littérature, et l'image idyllique du kolkhoze que présente Evrin est celle qu'il est politiquement correct de présenter. Bien des œuvres de l'époque, en effet, ont pour 
objectif de suivre la naissance des kolkhozes et leur pénétration dans la population ${ }^{13}$. Evrin se contente de suivre deux personnages emblématiques, incarnant les deux pôles idéologiques en conflit. Tyrpimij et Vasilij, le jeune Russe qui incarne les valeurs nouvelles, vont passer une semaine à la chasse ensemble. Ils se retrouvent en tête à tête dans la forêt et apprennent à se connaître et à se comprendre. Vasilij finira par se faire respecter du vieillard qui, découvrant d'un peu plus près les innovations qui lui sont proposées, choisit enfin, de son plein gré, de rejoindre le kolkhoze. Or la semaine de chasse est parsemée d'incidents et de tensions que les deux personnages finissent par dépasser pour arriver à s'entendre. C'est dans le traitement de ces contradictions qu'apparaît la maîtrise de la plume d'Evrin.

\section{Des conflits qui n'ont pas vieilli}

13 Le lecteur devine très vite l'issue "positive » du récit. Avouons aussi que l'enjeu l'adhésion du vieux Mansi au kolkhoze - vu rétrospectivement, à plus de soixante-dix ans de distance, a perdu de son actualité et de son intérêt. Pourtant, le récit demeure encore aujourd'hui non seulement lisible, mais également attachant. C'est qu'à côté du code idéologique - qui est dépassé -, il reste un problème qu'Evrin avait inscrit dans sa problématique principale, mais qui est suffisamment autonome pour susciter encore l'intérêt: l'opposition de deux cultures profondément différentes. Parce que le traitement de la confrontation culturelle n'était pas l'objectif affiché de l'auteur, mais un élément illustratif, il parvient à échapper au schématisme des déclarations plus directement liées à la politique de l'époque.

14 Nous pouvons identifier dans le récit deux lignes de contradictions illustratives qui attirent l'attention du lecteur: l'opposition des croyances et les contradictions interethniques aboutissant à la difficulté de dialoguer.

\section{L'opposition des croyances}

15 Incontestablement, nous avons là affaire à un domaine dans lequel le schématisme est un risque toujours présent. N'oublions pas le contexte dans lequel le récit a été écrit : à partir du tout début des années 1930, le combat contre les chamanes est engagé par le pouvoir de manière particulièrement violente ${ }^{14}$ : accusés en même temps de tromper et d'exploiter le peuple et de soutenir les riches éleveurs de rennes, assimilés aux koulaks, ils sont considérés comme ennemis de classe et systématiquement persécutés pendant toutes les années 1930.

Or la dimension religieuse constitue l'une des lignes directrices du récit. Dès l'introduction, le refus de Tyrpimij d'adhérer au kolkhoze est expliqué par ses «bizarreries»: «Il avait ses bizarreries, qui l'empêchaient d'adhérer au kolkhoze: Tyrpimij croyait en un dieu, il croyait aussi au chamane, il intervenait souvent contre les Russes ${ }^{15} »$.

Tyrpimij est donc "croyant». Sa religiosité est syncrétique, c'est un mélange de christianisme et d'animisme: il prie le soleil, mais il le fait en se signant et en murmurant des prières ${ }^{16}$. Il est incontestablement intéressant, ne serait-ce que d'un point de vue ethnographique, de relever la manière dont les deux systèmes religieux s'actualisent dans la pratique du vieux Mansi. Quand le premier élan est abattu, 
Tyrpimij veut prier. Quand Vasilij est sauvé d'une situation délicate, le vieux chasseur commente : "C'est Dieu qui t'a sauvé ». Quand le jeune Russe rapporte les oreilles de l'ours abattu, son compagnon est indigné : quel besoin avait-il d'humilier la bête abattue ? On voit ainsi que le lien avec la nature, le respect de ses éléments, sont des éléments qui demeurent dominants. Vasilij, selon les conceptions athées des communistes, rejette toute religion, tout rituel, toute croyance. Mais même là, Evrin fait preuve d'une finesse certaine : le discours de Vasilij n'est pas purement déclaratif, il tombe à propos et il est également justifié par l'expérience personnelle du jeune chasseur. Vasilij réagit toutes les fois que Tyrpimij se livre à des actes rituels; celui-ci pour sa part ne lui renvoie pas un contrediscours. Il répond de manières différentes : interrompu par le jeune Russe alors qu'il commençait à rendre grâces à Dieu pour la capture de l'élan, il lui exprime son mécontentement («si j'avais su, je ne serais pas parti avec toi dans la forêt $t^{17}$ )) puis tente de se justifier ("prie non point devant une icône, prie le soleil, le ciel ${ }^{18}$ !»). De manière générale d'ailleurs, c'est un silence perplexe qui répond au discours du chasseur russe.

Dans bien des textes de la même époque, les croyances chamaniques sont dénoncées avec bien plus de fermeté politique. C'est ainsi qu'en 1937, Ivan Nogo, un Nénètse du Yamal responsable du parti, écrit une pièce intitulée Le chamane ${ }^{19}$ qui montre le chamane sous un jour particulièrement sombre, et les gens qui s'adressent à lui comme des victimes. C'est aussi à la même époque que paraît à Leningrad un recueil de textes d'auteurs issus des peuples du Nord sous le titre Je ne crois pas aux chamanes ${ }^{20}$. Ici, le vieux Mansi attaché à ses croyances n'est montré ni comme une victime ni comme un naif crédule : il exprime simplement, sans exhibitionnisme, la sagesse traditionnelle de son peuple. Ses pratiques religieuses, présentées avec d'autant plus de distanciation qu'elles sont immédiatement contrées par les réactions de Vasilij, ne sont jamais réellement ridiculisées. Cette attitude est d'ailleurs exprimée par Vasilij lui-même, qui, vers la fin du récit, dans un dialogue où finalement les deux personnages parviennent à communiquer à cœur ouvert, proclame sa profession de foi : «Le parti communiste nous a conduits vers une vie meilleure, vers des jours radieux. Nous vivons maintenant d'après le bon sens et la raison, et nous conservons le passé en souvenir de nos aïeux ${ }^{21} »$. C'est en fait l'attitude que nous ressentons chez l'auteur : c'est du passé, mais nous le respectons en tant que tel. N'est-ce pas ainsi d'ailleurs qu'il nous faut interpréter le don fait par le jeune Mansi d'objets de culte au musée ethnographique?

\section{Les tensions interethniques}

Curieusement, chez Evrin, l'existence d'un conflit potentiel entre les autochtones et les Russes n'est pas un sujet tabou. Il se trouve même au cœur du récit. Cela est à noter, car ce n'est pas une occurrence fréquente dans les littératures des peuples de l'URSS à l'époque. Dans la région de la Volga, par exemple, les années trente sont marquées par le spectre du nationalisme, identifié dans toute référence à l'opposition des ethnies, à laquelle il était politiquement correct de substituer l'opposition de classes. Les procès dont les intellectuels nationaux sont victimes, commencés en 1932, se poursuivent et se multiplient aux alentours de 1937. Dans ce contexte, la voix d'Evrin est sainement rafraîchissante et rappelle par sa tonalité les positions qui avaient été officielles dans les années 1920. 
20 Parmi les bizarreries de Tyrpimij, en effet, il y a le fait qu'il était souvent amené à intervenir contre les Russes (p.52). Mais aussitôt Evrin en explique la raison: «À l'époque tsariste, lui, Mansi, avait eu plusieurs fois l'occasion de se quereller avec des marchands russes, qui tentaient de le duper ; depuis, il se méfiait de tous les Russes ${ }^{22}$ ". L'auteur suggère que la généralisation est abusive - mais son explication des raisons de la méfiance du vieux Mansi ressemble bien à une justification. Elle rappelle d'ailleurs les commentaires faits treize ans plus tôt par le ministre soviétique de l'Éducation luimême sur les relations interethniques dans le Nord :

Les petits peuples nomades du Nord, qui parlent différentes langues peu connues, éprouvent à l'égard de la culture russe une terreur qui va jusqu'à la haine. Cette haine a pris naissance dans l'impitoyable exploitation dont ils ont été victimes dans les temps passés. Le mot russe signifie dans le Nord " marchand " et « exploiteur ». Quand, naguère, les autochtones voyaient arriver la civilisation russe, ils s'enfuyaient pour lui échapper dans les ténèbres du cercle polaire. Il nous faut tenir compte de ce difficile héritage, de cette haine envers la culture - une haine qu'il n'est guère facile de dépasser. (Lunačarskij 1927, p. 18-19)

21 Ces préjugés vont être remis en cause tout au long du récit, grâce à la coexistence avec un Russe, personnage positif, qui a pour fonction de le faire changer d'avis. Mais attention, il ne s'agit pas de n'importe quel Russe : non seulement Vasilij fait partie des pauvres (orphelin, il a été pris en charge par un oncle pauvre et militant), mais il a épousé une Mansie et est allé vivre dans le village de sa femme. C'est donc un Russe qui a déjà fait un pas vers les Mansis. La dimension la plus intéressante dans la confrontation des deux personnages n'est en fait pas tant l'opposition idéologique l'ancien et le nouveau qui s'affrontent - que les difficultés de communication entre les deux personnages, difficultés qui tiennent moins à leurs individualités qu'aux malentendus de la confrontation interculturelle. Une partie importante des tensions dont les péripéties sont parsemées sont en effet dues à des incompréhensions mutuelles. Voyons plutôt.

Dès le premier jour Vasilij se vexe. La raison principale est qu'il ne comprend pas l'humour du vieux Mansi, un humour qu'il prend pour de l'agressivité. Les deux chasseurs n'ont pas trouvé de gibier, la journée a été mauvaise. Tyrpimij lance à Vasilij : « Tu ne portes pas chance. C'est à cause de toi que nous ne trouvons rien ${ }^{23}$ ». Vasilij se fâche (p. 53) : "il ne savait pas que Tyrpimij plaisantait ${ }^{24}$ ». C'est en effet là un type d'humour qui lui est étranger, alors même que toute personne étrangère qui séjourne chez les peuples du Nord doit être prête à accepter de devenir l'objet de plaisanteries ouvertes, qui ne préjugent en rien de la bienveillance de ses hôtes ${ }^{25}$.

La tension s'aggrave dans le courant de la journée, car Vasilij est fatigué et Tyrpimij continue à marcher. De plus, les chiens ont disparu, ce qui inquiète terriblement le chasseur russe. C'est la première fois qu'il chasse. Et il n'accepte pas l'imperturbabilité et l'optimisme du vieux Mansi. Entre-temps, celui-ci a eu, lui aussi, l'occasion de se vexer - l'insistance pessimiste du jeune Russe lui apparaît comme une manière de se moquer de lui : "Pourquoi donc montres-tu les dents? Moi je plaisante, et toi, tu te moques de $\operatorname{moi}^{26} \ldots$... (p. 56). Le vieux Mansi, du haut de son expérience de la chasse, est confiant, il sait que les chiens ne sont pas perdus. Le jeune Russe, au lieu de se montrer modeste et de lui faire confiance, se laisse emporter par ses craintes et fait fi de l'expérience de l'autochtone : "Cela fait quarante ans que tu chasses, alors que moi, je dors pour la première fois dans ce bois. Je pense qu'ils ne nous retrouveront $\operatorname{pas}^{27} »($ p. 56). Pire, il interprète la sérénité du Mansi à sa manière et selon des schémas 
préétablis qui font de l'autochtone le paresseux; il se dit : «Tyrpimij est venu dans la forêt non point pour chasser, mais pour se reposer dans la cabane de chasse et y manger du pain ${ }^{28} »($ p. 55).

Le deuxième jour, les choses se présentent mieux, les chasseurs parviennent à dénicher un couple d'élans. Mais Vasilij se laisse surprendre et rate sa proie. C'est dans ce contexte, alors qu'il s'apprête à écorcher la bête abattue par Tyrpimij, qu'il a une parole malencontreuse : «J'ai trente-cinq ans, mais je n'ai jamais vu un taureau pareil ${ }^{29} »$. Or Tyrpimij était appelé au village «le taureau ». Il réagit violemment, croyant que son compagnon se moque de lui : «Si je t'entends répéter ce mot, je te coupe la tête ${ }^{30}$ !» C'est le début d'une série d'incidents qui font croire au vieux Mansi que le jeune chasseur se moque de lui : il ne faut pas oublier dans ce contexte sa méfiance à l'égard de tous les Russes. Vasilij est troublé : «Il ne soutenait la conversation que mollement, de peur de dire quelque chose de travers ${ }^{31} »$. Dans les tensions de cette deuxième journée, les décalages culturels jouent un rôle moins direct que le pur hasard, mais la violence des réactions du vieux Mansi s'explique par ses préjugés de départ contre les Russes en général.

Le troisième jour est marqué par des épisodes qui auraient pu tourner au tragique, avec la rencontre des chasseurs avec les ours. L'inexpérience de Vasilij lui coûte cher : il se perd et ne reconnaît pas la présence de l'ours aux aboiements de ses chiens. Il est surpris par un deuxième ours après avoir abattu le premier, alors qu'il veut achever l'animal blessé. L'ours attaque de ses dernières forces Tyrpimij venu à la rescousse. Ainsi, les deux chasseurs se sauvent-ils en quelque sorte mutuellement. L'atmosphère entre eux est à la confiance. Celle-ci est tout juste entamée par la réaction de Tyrpimij à la vue des oreilles de l'ours. Pour lui, cet acte est une barbarie inutile et il s'empresse de procéder à un rituel pour demander pardon à l'ours. Et là, une fois de plus, l'opposition entre Mansis et Russes apparaît, amplifiée dans la prière de l'homme :

Mon petit ours, ma colombe, je ne t'ai pas fait de mal, j'ai toujours chassé avec mon arc et mes flèches. Nous, les Mansis, nous ne savons pas fabriquer les balles, nous n'avons pas de fusils. Ce sont les Russes qui les ont inventés et qui nous les vendent, à nous, les Mansis. Nous aussi, maintenant, nous utilisons les fusils en métal, mais nous n'oublions pas l'arc et les flèches. C'est avec eux que nous te prions. Grand ours, ne fais pas mon malheur. Je ne t'ai pas fait de mal. Ce sont les Russes, ce sont eux qui ont inventé les fusils en métal, qui s'en servent pour te tuer ${ }^{32} \ldots$

Vasilij ne se fâche pas, il réagit par l'humour, il réagit avant tout à la crainte du vieux Mansi que l'ours puisse lui tenir rigueur. Ainsi, la confrontation interethnique, qui aurait pu rebondir à ce point du récit, reste en suspens, car le Russe ne réagit pas à la mise en cause de son peuple en tant que tel. Et c'est ainsi que la tension retombe entre les deux hommes et que le préjugé antirusse de Tyrpimij s'estompe de lui-même.

\section{Les commentaires de la postérité}

À ma connaissance, ce texte n'a pas fait l'objet de commentaires ou d'études en dehors de la Russie ${ }^{33}$. Comme il ressort des pages qui précèdent, deux auteurs en Russie ont consacré des pages plus ou moins détaillées à l'auteur : il s'agit de la chercheuse mansie Kuzakova, apparentée à Evrin, et de Vjačeslav Ogryzko, dans la presse et dans son magistral répertoire des intellectuels des peuples du Nord. 

Naumova. Celle-ci caractérise l'œuvre par une seule notion : « une partie de la lutte du nouveau contre l'ancien " (Naumova 1940, p. 49). C'est dans la même perspective que je me suis livrée moi-même à un examen de quelques aspects de ce texte (Toulouze 1998, p. 124-125). article à l'autre, il ne change que de menus détails. De manière générale, il porte une appréciation positive sur ce texte :

Dans son récit Les deux chasseurs, on voit apparaître distinctement de nouveaux motifs, ancrés dans la réalité. L'auteur y montre l'amitié entre un vieux Mansi et un kolkhozien russe, la disparition de la méfiance envers les Russes, la conscience qui se libère des préjugés et des conceptions fausses. Le mérite artistique de cette œuvre tient à la justesse de la représentation de l'univers intérieur du héros et à la description animée de la chasse et de la taïga ${ }^{34}$. (Sergeev 1955, p. 176)

Nous constatons que cette présentation est très légèrement faussée : l'amitié n'est pas une donnée de départ, elle se forge en cours de route. C'est bien là qu'est le mérite de l'œuvre: elle est en fait moins déclarative que la lecture qui a pu en être faite. Concrètement, ce qui a changé entre le début de l'œuvre et la fin, c'est d'abord que Tyrpimij décide d'adhérer au kolkhoze, puis qu'entre les deux personnages se noue une amitié qui, très probablement, est amenée à déboucher sur l'affaiblissement, sinon la disparition, des préjugés antirusses. Mais le reste n'est pas si clair. Le vieil homme ne proclame pas son rejet de l'univers mental traditionnel de son peuple, il ne renie pas explicitement ses croyances. Il réfléchit. Il écoute ce que Vasilij a à lui dire, et médite. C'est d'ailleurs là l'un des mérites du récit: une «conversion » totale du vieux Mansi aurait été d'un schématisme trop frappant. Le commentaire de Sergeev fait dériver le récit sur cette pente. En 1952 son commentaire était identique à un détail près : les « conceptions fausses » étaient explicitement des « conceptions religieuses ${ }^{35}$ " (Sergeev 1952, p. 159). En 1956, le texte de 1955 n'a connu qu'un ajout: «l'amitié qui se noue entre un Mansi arriéré et un kolkhozien russe » (Sergeev 1956, p. 140). Il est curieux de retrouver exactement les mêmes thèmes, et notamment cette même formulation, douze ans plus tard chez Polonskij, qui s'exprime ainsi :

L'auteur relate chaleureusement l'amitié surgie entre un chasseur mansi arriéré et un kolkhozien russe, la manière dont la méfiance envers le Russe s'efface dans l'âme du Mansi. Le récit est attachant également par la profondeur de la représentation du monde intérieur des héros et par les tableaux poétiques, imprégnés d'amour, de la taïga chère à l'auteur ${ }^{36}$. (Polonskij 1967, p. 6)

Comme nous le voyons, les thèmes sont les mêmes. Les « préjugés » et «les conceptions fausses » de Sergeev ont disparu, mais le héros mansi est encore qualifié d'arriéré. Là aussi, nous avons affaire à un léger décalage dans le message de l'original : c'est avec beaucoup de délicatesse et de respect, sans jamais vraiment les ridiculiser, qu'Evrin présente les croyances de Tyrpimij. Les qualités littéraires de l'œuvre, pour Sergeev et pour Polonskij, sont identiques. Cette coïncidence ne manque pas de susciter des questions sur l'originalité de la vision de Polonskij. En tout cas, ce texte est repris ipsis verbis dans une publication de 1996 (Polonskij 1996, p. 151).

Voskobojnikov, enseignant à la Faculté des peuples du Nord, mentionne lui aussi, dans un écrit du milieu des années 1950, le récit d'Evrin et le résume en disant que «le vieux Mansi lie son sort au héros du récit, un chasseur russe ${ }^{37}$ (Voskobojnikov 1956, p. 43).

Études finno-ougriennes, 43 | 2011 
Outre qu'il est incorrect de présenter Vasilij comme un chasseur (c'était la première fois qu'il allait dans la forêt), en faire le héros du récit est également un abus : chez Evrin, l'attention est équitablement partagée entre les deux personnages, comme d'ailleurs le titre l'indique. D'ailleurs, s'il fallait faire ressortir l'un des deux personnages, c'est plutôt sur Tyrpimij que le lecteur s'arrête, car il est amené à évoluer de manière plus claire que son compagnon. Ce dernier en vient sans doute à davantage respecter son compagnon mansi et ses savoir-faire de chasseur qu'il ne le faisait au début. Mais le vieux Mansi, lui, prend une décision qu'il avait jusqu'alors refusé de prendre.

Nous voyons donc que les critiques, dans la décennie d'après-guerre et encore dans les années 1960, sont amenées à légèrement infléchir le message du récit pour en tirer avant tout un message de type politique: l'essentiel était de mettre en évidence l'émergence de la concorde entre les peuples. Le relatif équilibre qui existe dans le texte entre les personnages est effacé au profit du schéma qui fait de l'un le progressiste et de l'autre celui qui est mis sur le droit chemin. Dans les années 1970, la critique devient plus proche du texte, plus rigoureuse, plus intéressante aussi. Nous avons par exemple un paragraphe entier consacré par Komanovskij à ce récit, qui montre que le critique l'a lu attentivement. En effet, celui-ci voit désormais Tyrpimij autrement : «L'auteur a su brosser un portrait animé du vieux Mansi, avec ses préjugés, son savoir-faire de chasseur et son intelligence indépendante ${ }^{38} »$ (Komanovskij 1977, p.65). Ici, nous retrouvons le personnage attachant, complexe, du texte original. La critique s'est affranchie de la tyrannie du message. Il est vrai que celui-ci est moins d'actualité.

À la fin des années 1990, Ogryzko, qui consacre à Čejmetov une page et demie de son répertoire des écrivains, met en évidence des aspects quelque peu différents. Dire que "des gens de la taïga comparent leur vie avant et après la révolution d'octobre " (Ogryzko 1998, p. 397) est une généralisation quelque peu exagérée. Si, dans les grandes lignes, à un haut niveau d'abstraction, c'est bien de cela qu'il est question, la manière dont cette comparaison se fait est loin d'être directe. Le choix du kolkhoze ne se fait pas tant sur la base d'un bilan des avantages et des inconvénients, que sur le déclic créé par la toute nouvelle amitié avec Vasilij : d'ailleurs, au début du récit, Tyrpymyi n'était pas hostile, il était hésitant. Pour Ogryzko, la nouveauté tient dans le choix par l'auteur de héros d'appartenance ethnique différente, « chacun d'entre eux se distinguant par une vision du monde particulière ${ }^{39} "$ (ibid.). Ce qui précède montre bien que ma vision rejoint sur ce point celle d'Ogryzko, car c'est réellement dans la présentation côte à côte de deux mentalités différentes que réside l'intérêt du récit, voire son actualité puisque les contacts entre les deux communautés n'ont pas cessé, bien au contraire, et que les difficultés du genre de celles que rencontrent les deux personnages de ce récit peuvent très bien survenir à l'heure actuelle.

\section{Conclusion}

Cette œuvre méritait d'être sortie des oubliettes. Comme j'ai essayé de le montrer, elle présente une fraîcheur et une originalité qu'il faut souligner dans le contexte littéraire des années d'avant-guerre. Elle échappe au carcan du réalisme socialiste avant tout par son manque de totalitarisme dans la conception du socialisme. Les apparences sont sauves, et sans doute pour Evrin ne s'agissait-il pas seulement d'apparences: le vieux 
Mansi finit par adhérer au kolkhoze et je ne doute pas que l'auteur de ce récit soit sincèrement convaincu, au moment où il écrit, que, pour les Mansis, adhérer aux kolkhozes est la meilleure solution. Mais son œuvre n'est pas une œuvre militante et déclarative: elle laisse aux personnages et à leurs pensées de l'espace pour se rencontrer et pour s'ajuster. Cette œuvre se veut réaliste. Dans la réalité, les peuples du Nord n'auront pas longtemps l'espace nécessaire pour s'ajuster et l'étau se resserrera très vite, leur interdisant de rester ce qu'ils sont.

De toutes les œuvres des débuts des littératures des Ougriens de l'ob et des Samoyèdes, nous avons là, à mon sens, un texte qui garde encore aujourd'hui des qualités remarquables. Il serait dommage de le laisser plus longtemps dans l'oubli.

\section{BIBLIOGRAPHIE}

KÁLMÁN Béla, 1962, »Über die Wogulische Schriftsprache«, Ural-Altaische Jahrbücher, Band XXXIV, Heft 1-4, pp. 128-133.

KOMANOVSKIJ 1977 = КОМАНОВСКИЙ, Б.Л, ПУТИ РАЗВИТИЯ ЛИТЕРАТУР НАРОДОВ КРАЙНЕГО СЕВЕРА И ДАЛЬНЕГО ВОСТОКА СССР, МАГАДАН.

KUZAKOVA 1997 = КУЗАКОВА, ЕВДОКИЯ, «МАНСИЙСКИЙ ПИСАТЕЛЬ ЧЕЙМАТОВ (ПРЕДИСЛОВИЕ К РАССКАЗУ «ДВА ОХОТНИКА»)», ЮГРА 7/1997, С. 30.

LAMBERT Jean Luc, 2008, « De l'Évangile à l'ours en Russie impériale : Comment faire prêter serment à des peuples animistes? ", Revue d'études mongoles et sibériennes, $\mathrm{n}^{\circ}$ 38-39, p. 19-43.

LUNAČARSKIJ 1927 = ЛУНАЧАРСКИЙ А. В., «ЗАДАЧИ НАРКОМПРОСА НА КРАЙНЕМ СЕВЕРЕ», СЕВЕРНАЯ АЗИЯ. ОБЩЕСТВЕННО-НАУЧНЫЙ ЖУРНАЛ, КНИГА ТРЕТЬЯ, МОСКВА, С. 18-22.

NAUMOVA 1940 = НАУМОВА, В., (ПРЕДИСЛОВИе) ВОРЫЯП ХУМЫЙ, ЛЕНИНГРАД, С.49-50.

OGRYZKO 1997 = ОГРЫЗКО, ВЯЧЕСЛАВ ВЯЧЕСЛАВОВИЧ, «НЕУДАЧНЫЙ ВЫСТРЕЛ», ЛИТЕРАТУРНАЯ Россия, 31/1/1997 (Под ФАМ : В. КалМЫКОВ).

OGRYZКO 2002 = ОГРЫЗКО, ВЯЧЕСЛАВ ВЯЧЕСЛАВОВИЧ, «ЛИТЕРАТУРА ОТЧАЯНИЯ И НАДЕЖДЫ», ХАНТЫЙСКАЯ ЛИТЕРАТУРА, МОСКВА, С. 3-29.

POLONSKIJ 1967 = ПОЛОНСКИЙ, ЛАЗАРЬ ВУЛЬФОВИЧ, ЛИТЕРАТУРЫ, РОЖДЕННЫЕ ОКТЯБРЕМ (О ТВОРЧЕСТВЕ ПИСАТЕЛЕЙ ОБСКОГО СЕВЕРА), ТЮМЕНЬ.

POLONSKIJ 1996 = ПОЛОНСКИЙ, ЛАЗАРЬ ВУЛЬФОВИЧ, «ЛИТЕРАТУРа НАРОДОВ СЕВЕРА (В СОКРАЩЕНИИ)», КОСМОС СЕВЕРА, ТЮМЕНЬ, С. 151-170.

SERGEEV 1952 = СЕРГЕЕВ, М.А., «ЛИТЕРАТУРа НАРОДОВ СЕВЕРА», СИБИРСКИЕ ОГНИ, 1952/2 (9/10), C. $155-166$.

SERGEEV 1955 = СЕРГЕЕВ, М.А., «ЛИТЕРАТУРНОЕ ТВОРЧЕСТВО НАРОДОВ СЕВЕРА», СОВЕТСКАЯ ЭТНОГРАФИЯ, 1955/3, С. 173-187. 
SERGEEV 1956 = СЕРГЕЕВ, М.А., « ЛИТЕРАТУРа НАРОДОВ СЕВЕРА », ЛИТЕРАТУРа НАРОДОВ СИБИРИ, НОвОСИБИРск, с. 131-170.

SUSLOV 1931 = СУСЛОВ, И. М., «ШАМАНСТВО И БОРЬБА С НИМ», СОВЕТСКИЙ СЕВЕР, 1931/3-4, C. 89-152.

Toulouze Eva, 1996, « Les peuples du Nord et l'enjeu de la littérature », Mélanges offerts à M. de Labriolle, Slovo, $\mathrm{n}^{\circ} 17$, Paris, p. 301-315

TOUlouZE Eva, 1998 = ТУЛУЗ ЕВА, «СТАРОЕ И НОВОЕ В ПРОИЗВЕДЕНИЯХ ЛИТЕРАТОРОВ УРАЛЬСКИХ НАРОДОВ СЕВЕРА : ИДЕОЛОГИЯ СОЦИАЛИЗМА И ТРАДИЦИОННОЕ МИРОВОЗЗРЕНИЕ», АРТ 4/1998, СЫКТЫВКАР, С. 120-125.

VOSКOBOJNIKOV 1956 = ВОСКОБОЙНИКОВ, М.Г., «ЛИТЕРАТУРа НАРОДОВ КРАЙНЕГО СЕВЕРА ЗА 25 ЛЕТ (КРАТКИЙ ОБЗОР)», В ПОМОЩИ УЧИТЕЛЬЮ ШКОЛ КРАЙНЕГО СЕВЕРА, ВЫП. 6, 1956, С. 26-47.

\section{NOTES}

1. Les Mansis font partie de ce qui est appelé en Russie les peuples dits « peu nombreux» (en russe : мАлочисленный). Dans mon usage, le terme de «petit » n'a rien de dépréciatif : il rend compte exclusivement du faible nombre aussi bien de personnes s'en réclamant que de locuteurs de la langue, ce qui a un certain nombre de conséquences au quotidien.

2. 12269 personnes au recensement de 2010 .

3. En traduction russe : «НА БЕРЕГУ МАЛОЙ ЮКОНДЫ» (Sur les berges de la Petite Jukonda) ТВОРЧЕСТВО НАРОДОВ СЕВЕРА - ЛЕНИНГРАД 1954, avec aussi des rééditions plus tardives. Le texte a bien été écrit en mansi, mais il n'a été publié qu'en russe, dans une traduction de G. Gor.

4. D'après d'autres auteurs, Čejmatov, ou encore Čajmatov (Sergeev 1955, p. 176).

5. Mot formé à partir du toponyme Evra et signifiant, en russe, « celui d'Evra ».

6. En mansi et en russe : ВОРЫЯП ХУМЫЙ / ДВА ОХОТНИКа, ЛЕНИНГРАД, 1940.

7. ТУНДРА И ТАЙГА.

8. О НАШЕЙ ЖИЗНИ.

9. “НА ОСТРОВЕ” - НАША СТРАНА 1937. Texte écrit en nénètse et traduit en russe par G. Verbov.

10. “КАК МЫ ЖИЛИ ПРИ ЦАРЕ” - СОВЕТСКАЯ АРКТИКА 1937/11.

11. МЛАДШИЙ СЫН ВЭДО, ЛЕНИНГРАД, 1960. Le texte a été écrit en fait en 1940 et a obtenu la même année un prix au concours des œuvres des écrivains du Nord.

12. Trofim dans la traduction russe.

13. C'est par exemple le cas dans la littérature oudmourte de la trilogie de G. Medvedev Le champ de Lozja, en russe лозя БЕСмен, dont le dernier volet a été achevé peu avant l'arrestation de l'écrivain, en 1937.

14. Le cadre idéologique de cette lutte apparaît clairement dans un article programmatique de Suslov en 1931 (Suslov 1931).

15. «БЫЛИ У НЕГО СВОИ СТРАННОСТИ, КОТОРЫЕ МЕШАЛИ ЕМУ ВОЙТИ В КОЛХОЗ. ТРОФИМ ВЕРИЛ В БОГА, ВЕРИЛ И ШАМАНУ, НЕРЕДКО ВЫСТУПАЛ ПРОТИВ РУССКИХ» (р. 52).

16. «С ЭТИМИ СЛОВАМИ САМ НАЧАЛ БЫСТРО КРЕСТИТЬСЯ И БОРМОТАТЬ МОЛИТВЫ» (р. 60).

17. «ЗНАЛ БЫ Я, ТАК И В ЛЕС БЫ С ТОБОЙ НЕ ПОШЕЛ» (р. 61).

18. «МОЛИСЬ НЕ НА ИКОНУ, а НА СОЛНЦЕ, НА НЕБО» (p. 61).

19. En nénètse: Tadebe. La pièce a été éditée en nénètse, à l'époque où l'alphabet latin était encore de rigueur. Elle s'accompagne d'une traduction russe due à G. Verbov.

20. НЕ ВЕРЮ ШАМАНАМ, ЛЕНИНГРАД, 1939. 
21. «НАС КОММУНИСТИЧЕСКАЯ ПАРТИЯ К ХОРОШЕЙ ЖИЗНИ, К СВЕТЛЫМ ДНЯМ ПРИВЕЛА. МЫ ЖИВЕМ ТЕПЕРЬ ЗДРАВЫМ УМОМ, А ПРОШЛОЕ ХРАНИМ КАК ПАМЯТЬ НАШИХ ПРЕДКОВ».

22. «В ЦАРСКОЕ ВРЕМЯ ОН, МАНСИ, НЕ РАЗ ССОРИЛСЯ С РУССКИМИ ТОРГОВЦАМИ, СТАРВШИМИСЯ ЕГО НАДУТЬ ; С ТЕХ ПОР ОН НА ВСЕХ РУССКИХ СТАЛ СМОТРЕТЬ С НЕДОВЕРИЕМ».

23. «НЕУДаЧЛИВЫЙ ТЫ ЧЕЛОВЕК. ИЗ-ЗА ТЕБЯ НЕ МОЖЕМ НИЧЕГО НАЙТИ».

24. «ОН НЕ ЗНАЛ, ЧТО ТРОФИМ ШУТИТ».

25. C'est là mon expérience personnelle de terrain, entièrement partagée par tous mes collègues anthropologues ayant travaillé chez les peuples du Nord de la Russie (informations orales de Laur Vallikivi, Liivo Niglas etc).

26. «ДА ЧТО ТЫ ЗУБЫ-ТО СКАЛИШЬ, Я ШУТЯ ГОВОРЮ, А ТЫ ИЗДЕВАЕШЬСЯ.»

27. «ТЫ СОРОК ЛЕТ ОХОТИШЬСЯ, А Я ПЕРВЫЙ РАЗ СПЛЯ В ТАКОМ ЛЕСУ. Я ДУМАЮ, ЧТО ОНИ НЕ НАЙДУТ НАС».

28. «ТРОФИМ (...) ПРИШЕЛ В ЛЕС НЕ ОХОТИТЬСЯ, А В ИЗБУШКЕ ЛЕЖАТЬ ДА ХЛЕБ ЕСТЬ».

29. «ТРИДЦАТЬ ПЯТЬ ЛЕТ ПРОЖИЛ НА СВЕТЕ, А ТАКОГО БЫКА НЕ ВИДАЛ» (p. 62). Pour la clarté de la situation, précisons que le terme équivalent au français taureau est utilisé en russe aussi pour désigner les mâles des cervidés.

30. «ТОЛЬКО ЕШЁ РАЗ УСЛЫШУ, ГОЛОВУ ТЕБЕ СРУБЛЮ» (р. 62).

31. «ВАСИЛИЙ ВЯЛО ПОДДЕРЖИВАЛ РАЗГОВОР, ОПАСАЯСЬ, КАК БЫ НЕ СКАЗАТЬ ЧТО-ЛИБО НЕВПОПАД» (р. 63).

32. «МЕДВЕДУШКА, ГОЛУБЧИК, Я ТЕБЕ НЕ ДЕЛАЛ НИКАКОГО ЗЛА, ОХОТИЛСЯ ВСЕГДА СО СТРЕЛАМИ И ЛУКОМ. МЫ, МАНСИ, НЕ УМЕЕМ ДЕЛАТЬ ПУЛЬ, НЕ ИМЕЕМ РУЖЕЙ. ВЫДУМАЛИ ИХ РУССКИЕ И ПРОДАЮТ НАМ, МАНСИ. ТЕПЕРЬ МЫ ТОЖЕ ХОДИМ С ЖЕЛЕЗНЫМИ РУЖЬЯМИ, НО НЕ ЗАБЫВАЕМ И ЛУКА СО СТРЕЛОЙ. СО СТРЕЛАМИ И ЛУКОМ МЫ МОЛИМСЯ ТЕБЕ. ВЕЛИКИЙ МЕДВЕДУШКА, НЕ ВВЕДИ МЕНЯ К БЕДУ. Я НЕ ДЕЛАЛ ТЕБЕ ЗЛА. ЭТО ВСЕ РУССКИЕ, ОНИ ВЫДУМАЛИ ЖЕЛЕЗНЫЕ РУЖЬЯ, ОНИ НАДУМАЛИ УБИВАТЬ ТЕБЯ ИМИ» (pp. 70-71). Il s'agit là d'une formule rituelle, que nous retrouvons souvent dans les textes de prières. Ici, Evrin reproduit des formules qu'il a certainement entendues (cf. Lambert 2008).

33. À l'exception d'un bref passage dans une de mes études (Toulouze 1998, p. 124-125).

34. «В ЕГО ПОВЕСТИ ОТЧЕТЛИВО ПРОЗВУЧАЛИ НОВЫЕ МОТИВЫ, РОЖДЕННЫЕ ДЕЙСТВИТЕЛЬНОСТЬЮ. АВТОР ПОКАЗАЛ ДРУЖБУ СТАРИКА МАНСИ С РУССКИМ КОЛХОЗНИКОМ, ИЗЧЕЗНОВЕНИЕ НЕДОВЕРИЯ К РУССКИМ, ОСВОБОЖДЕНИЕ СОЗНАНИЯ ОТ ПРЕДРАССУДКОВ И ЛОЖНЫХ ПРЕДСТАВЛЕНИЙ. ХУДОЖЕСТВЕННЫЕ ДОСТОИНСТВА ПРОИЗВЕДЕНИЯ ЗАКЛЮЧАЮТСЯ В ПРАВДИВОМ ИЗОБРАЖЕНИИ ВНУТРЕННОГО МИРА ГЕРОЯ И ЖИВОМ ОПИСАНИИ ТАЙГИ И ОХОТЫ.» 35. «ЛОЖНЫХ РЕЛИГИОЗНЫХ ПРЕДСТАВЛЕНИЙ».

36. «АВТОР ТЕПЛО ПОВЕДАЛ О ДРУЖБЕ, ВОЗНИКШЕЙ МЕЖДУ ОТСТАЛЫМ МАНСИЙСКИМ ОХОТНИКОМ И РУССКИМ КОЛХОЗНИКОМ, О ТОМ, КАК ПРЕОДОЛЕВАЕТСЯ В ДУШЕ МАНСИ ЧУВСТВО НЕДОВЕРИЯ К РУССКОМУ ЧЕЛОВЕКУ. РАССКАЗ ПРИВЛЕКАЕТ И ГЛУБОКИМ РАСКРЫТИЕМ ВНУТРЕННОГО МИРА ГЕРОЕВ И ПОЛНЫМИ ЛЮБВИ ПОЭТИЧЕСКИМИ КАРТИНАМИ РОДНОЙ ТАЙГИ.» 37. «СТАРИК-МАНСИ СВЯЗЫВАЕТ СВОЮ СУДЬБУ С ГЕРОЕМ РАССКАЗА, РУССКИМ ОХОТНИКОМ». 38. «АВТОР СУМЕЛ ОЧЕРТИТЬ ЖИВУЮ ФИГУРУ СТАРОГО МАНСИ С ЕГО ПРЕДРАССУДКАМИ, ОХОТНИЧЬЕЙ СНОРОВКОЙ И САМОСТОЯТЕЛЬНЫМ УМОМ».

39. «КАЖДЫЙ ИЗ КОТОРЫХ ОТЛИЧАЛСЯ ОСОБЫМ МИРОВОСПРИЯТИЕМ». 


\section{RÉSUMÉS}

Les premières œuvres des écrivains débutants issus des peuples du Nord ont été injustement oubliées : écrites dans la deuxième moitié des années 1930, elles étaient, certes, naïves et peu sophistiquées, mais exploraient un terrain nouveau. Il est vrai que la guerre, survenue peu après, a balayé ces timides débuts, d'autant que les jeunes auteurs, presque sans exception, ont été emportés dans le massacre. Parmi les œuvres qui méritent d'être sorties de l'oubli, on trouve Deux chasseurs, de Pantelejmon Evrin, première œuvre en prose de la littérature mansie (1940). Curieusement pour l'époque, Evrin aborde deux thèmes délicats : les tensions entre autochtones et Russes, et le heurt du spirituel et du profane. Cet article se propose de faire le point sur ce que nous savons de cet auteur mansi, d'analyser ces thèmes tels qu'ils ont été traités dans ce récit, et de donner un aperçu de la réception de ce récit par la critique.

The first texts by Russia's Northern indigenous authors have been unjustly forgotten: they were written in the second half on the 1930s, were naive and not very sophisticated, but they opened new ways. Indeed, the oncoming war swept away these debuts, while the young authors, almost without exception, disappeared in the turmoil. Among the literary works that deserve to be remembered there is Pantelejmon Evrin's Two Hunters, the starting point of Mansi literature (1940). Curiously enough, Evrin touches upon two delicate issues: tensions between natives and Russians, and the confrontation between beliefs and socialist materialism. This article reviews what is known about the Mansi author, analyses the issues as they are presented in the short novel and gives an overview of its reception.

Põhja-Venemaa põliselanikest kirjanike esimesed teosed on põhjendamatult unustusse vajunud: need kirjutati 1930. aastate teises pooles, tekstid olid naiivsed ja mitte väga keerukad, kuid rajasid uusi lähenemissuundi. Kahtlemata unustati need teosed vähemalt osaliselt sõja tõttu, mis puhkes varsti pärast nende avaldamist; ka autorid hukkusid pea eranditult. Need teosed ja autorid väärivad siiski mäletamist. Üks neist on Panteleimon Jevrin ja tema Kaks jahimeest (1940), mida loetakse mansi kirjanduse esimeseks teoseks. Jevrin käsitleb oma jutustuses kaht rasket küsimust: ühelt poolt pingeid põliselanike ja venelaste vahel, teiselt poolt kohalike usundite ja sotsialistliku materialismi kokkupõrget. Artiklis uurib autor, mida sellest mansi kirjanikust teatakse, analüüsib jutustuse teemasid ning kommenteerib teose retseptsiooni.

\section{INDEX}

Mots-clés : Intelligentsia, fiction, Kolkhoze, tensions interethniques, pratiques religieuses, Koulak, Christianisme, Mansis, réalisme socialiste, Oudmourtes, chasse, Nénetse, animisme, Petit peuple, Peuples du Nord

Index chronologique : XXe siècle (début), XXe siècle

motscleset fiktsioon, rahvustevahelised pinged, usuline tegevus, ristiusk, sotsialistlik realism, küttimine, animism, Lääne-Siber, mansi kirjandus

Thèmes : littérature

nomsmotscles Mansis

Index géographique : Sibérie occidentale

Keywords : fiction, interethnic tensions, religious practice, Christianity, Socialist realism, hunting, animism, Western Siberia, Mansi literature 
AUTEUR

EVA TOULOUZE

Maître de conférences habilité

Inalco

Chercheuse rattachée à l'Université de Tartu 